\title{
A Readout Driver for the ATLAS LAr-Calorimeter at a High Luminosity LHC
}
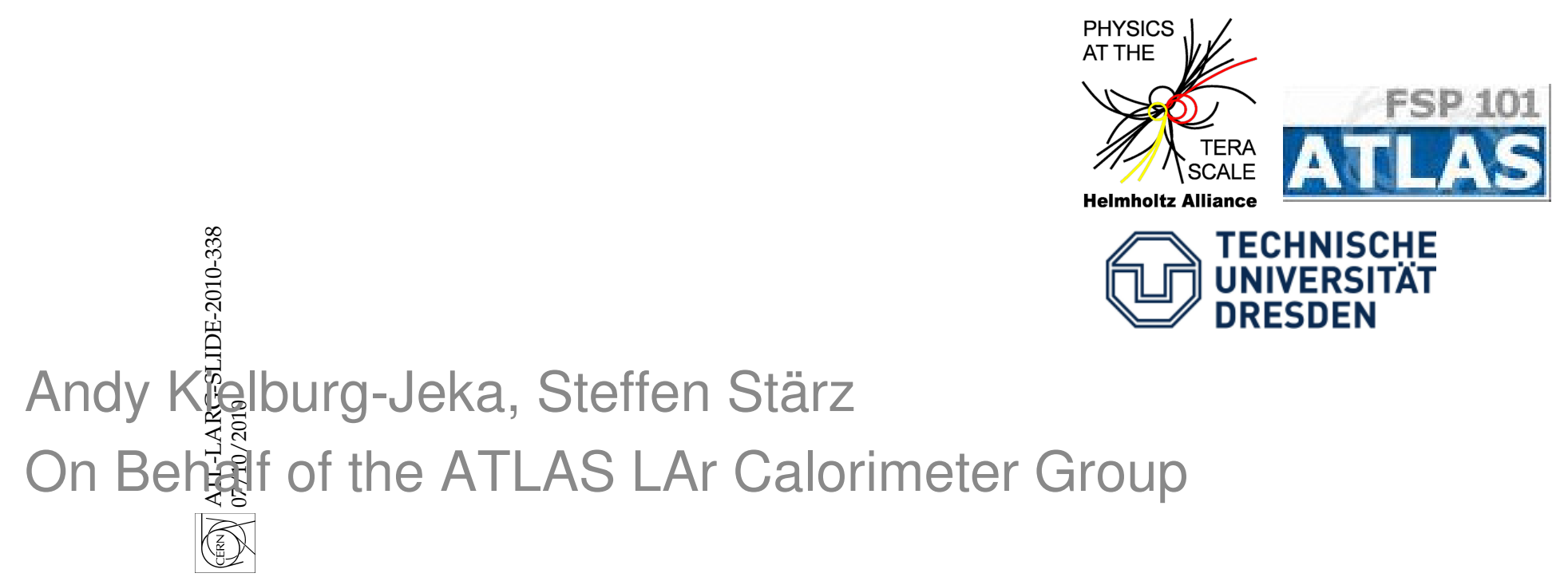

TECHNISCHE DRESDEN 


\section{Outline}

- ATLAS detector and current LAr calorimeter read-out system

- upgrade to HL-LHC

- readout scheme for HL-LHC

- hardware development status

- summary and outlook 


\section{The ATLAS Detector}

- 4 high granularity LAr calorimeters
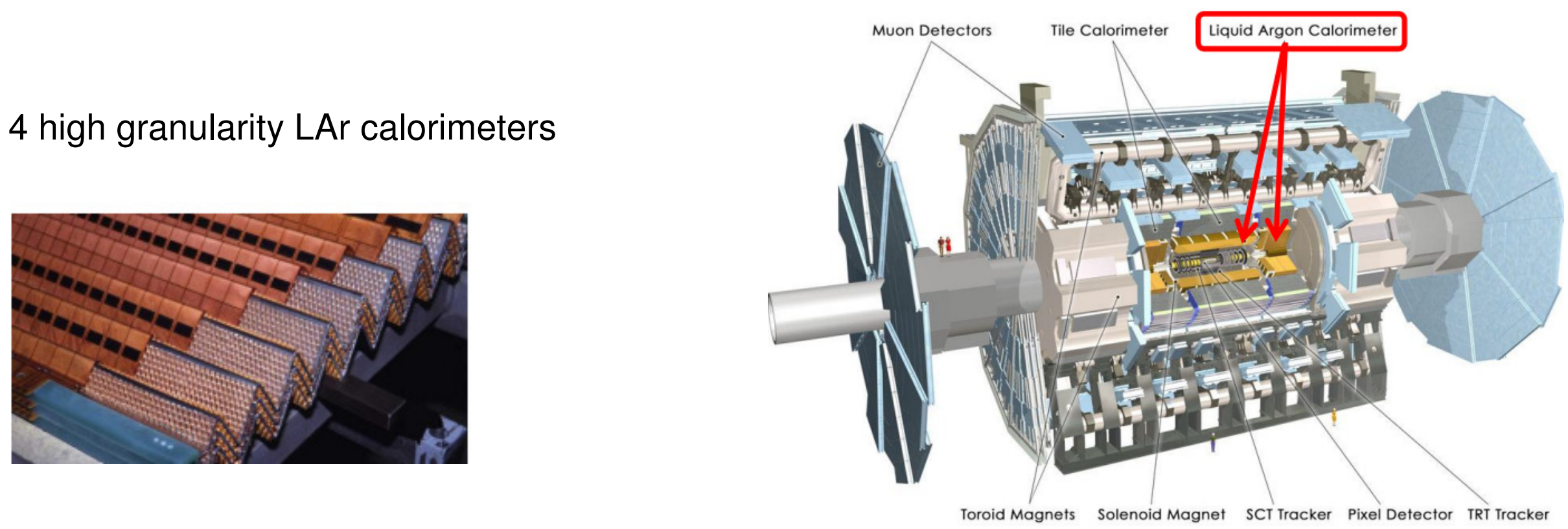

- 182486 readout channels

- $40 \mathrm{MHz}$ proton-proton collision rate

- front-end and trigger-sum electronics

- mounted on detector,

- operates in radiation environment

- back-end electronics and additional trigger logic - in counting room outside the experimental cavern

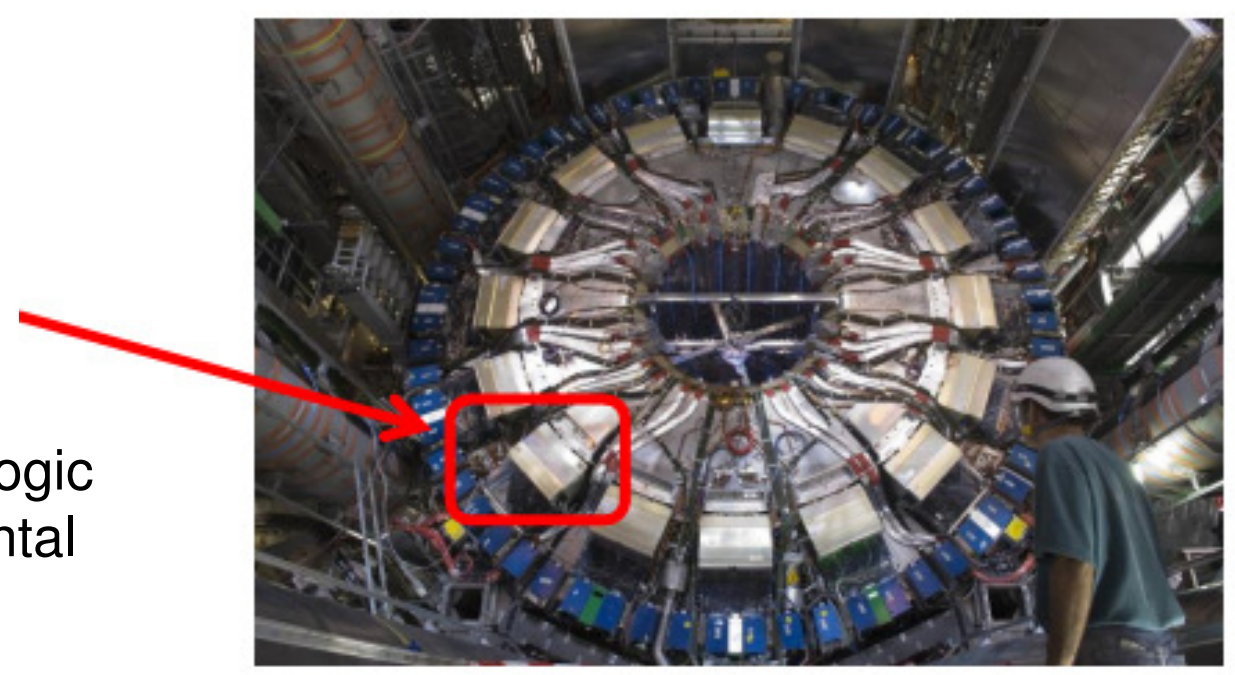




\section{Current LAr Calorimeter Readout System}

-1524 front-end boards (FEB)

- up to 128 channels

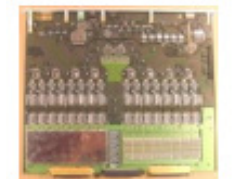

- preamp, pulse shaping, buffer and sampling

- analog signal to L1 calorimeter trigger (@ 40MHz)

- readout clock is synchronous with LHC bunchcrossing (25 ns)

- connected to off-detector electronics by

- 58 copper trigger cables

- 1600 optical links

- 192 read-out driver boards (ROD)

- digital filter

- data transferred only after L1 accept @ 100kHz

- 800 optical links to DAQ PCs
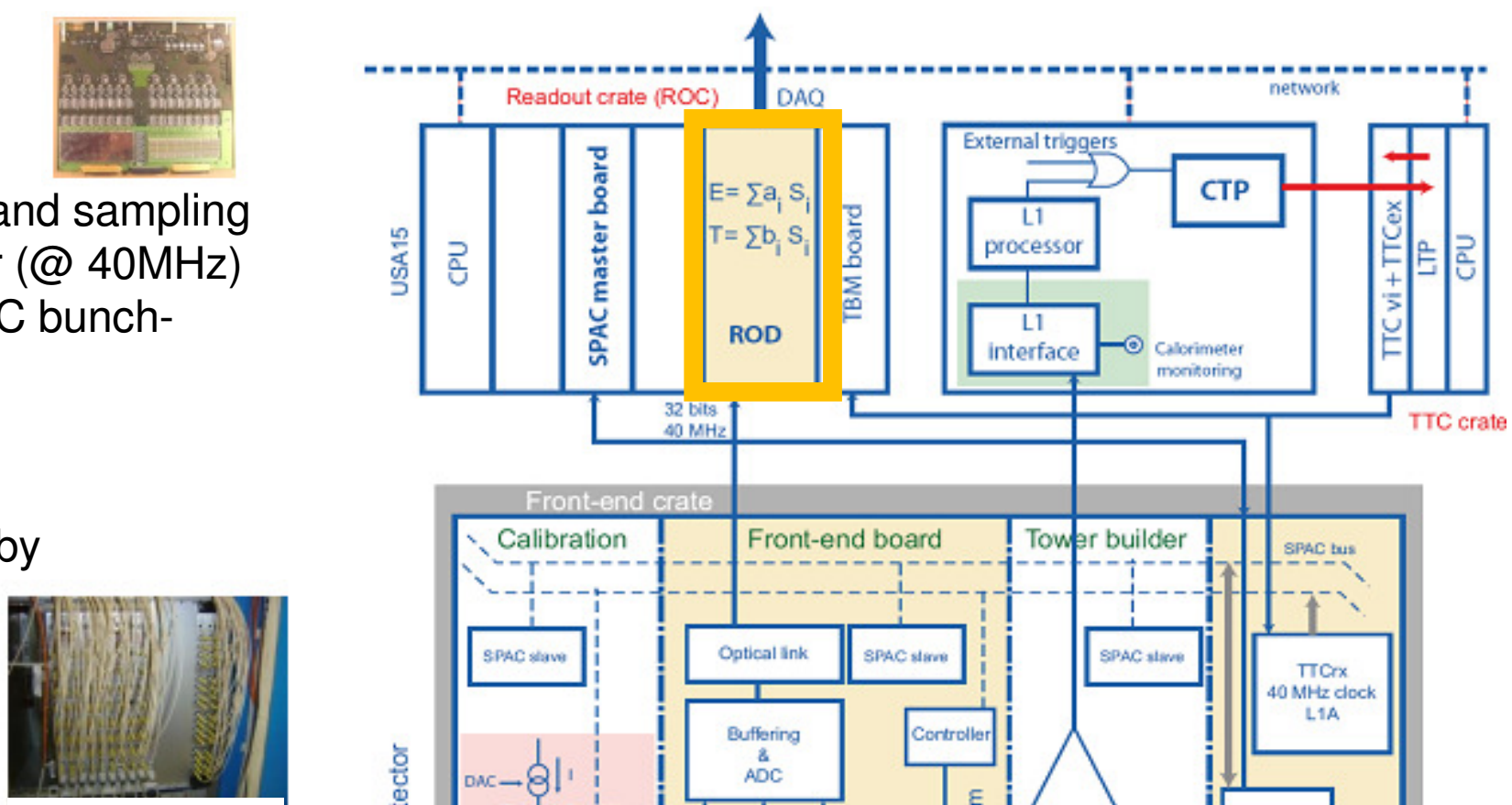

- 68 read-out buffer PCs (ROB)

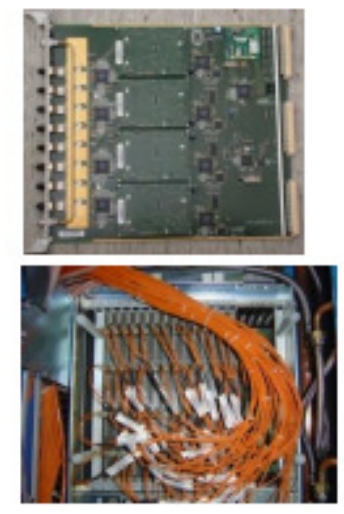

- DAQ and high-level trigger buffer

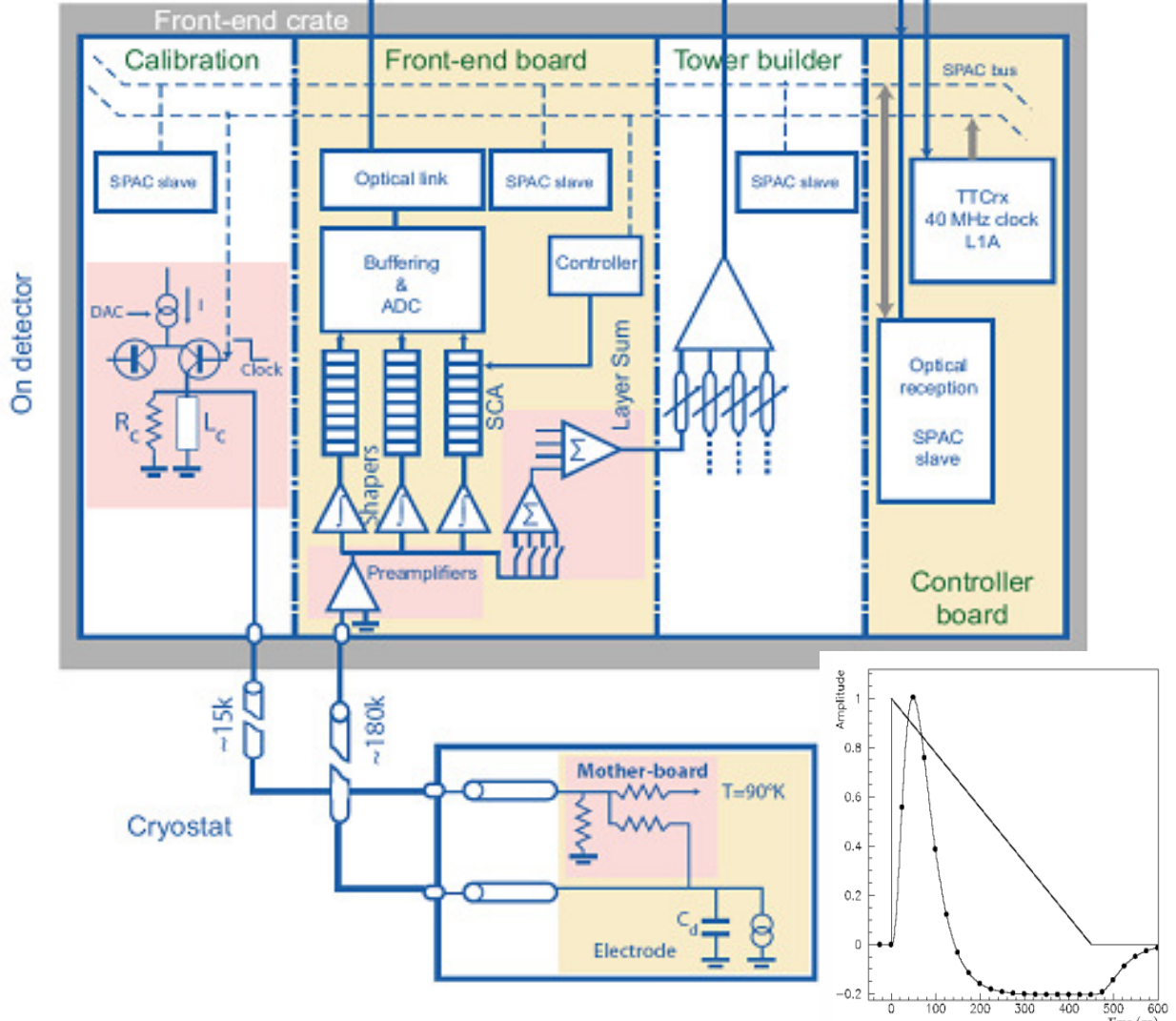




\section{Upgrade to High Luminosity LHC}

- Several reasons for upgrade:

- radiation tolerance of front-end electronics: HL-LHC reaches up to $3000 \mathrm{fb}^{-1}$

- qualified for $\sim 700 \mathrm{fb}^{-1}$ (+ safety margin)

$\rightarrow$ not possible to operate hardware until end of HL-LHC

- ageing / reliability concerns of current electronics:

- some parts exceed 20 years age at HL-LHC start

- limited size of on-detector data buffers:

- current readout architecture does not allow increase in L1 latency nor bandwidth

- if readout is upgraded $\rightarrow$ chance to increase granularity of trigger input

- HL-LHC challenges:

$\sim 5 x$ higher radiation levels for on-detector electronics

$\sim 5 x$ higher total ionization dose for on-detector electronics

$\sim 5 x$ more simultaneous proton-proton interactions and pile-up background 


\section{Trigger Latency Concerns}

- Current LAr latency:

- 96 bunch-crossings (BC)

- +10 reserve BC

- Scenario with new trigger design:

- example: modified muon trigger

- trigger violates the LAr latency by $6.5 \mathrm{BC}$

- would create dead-time
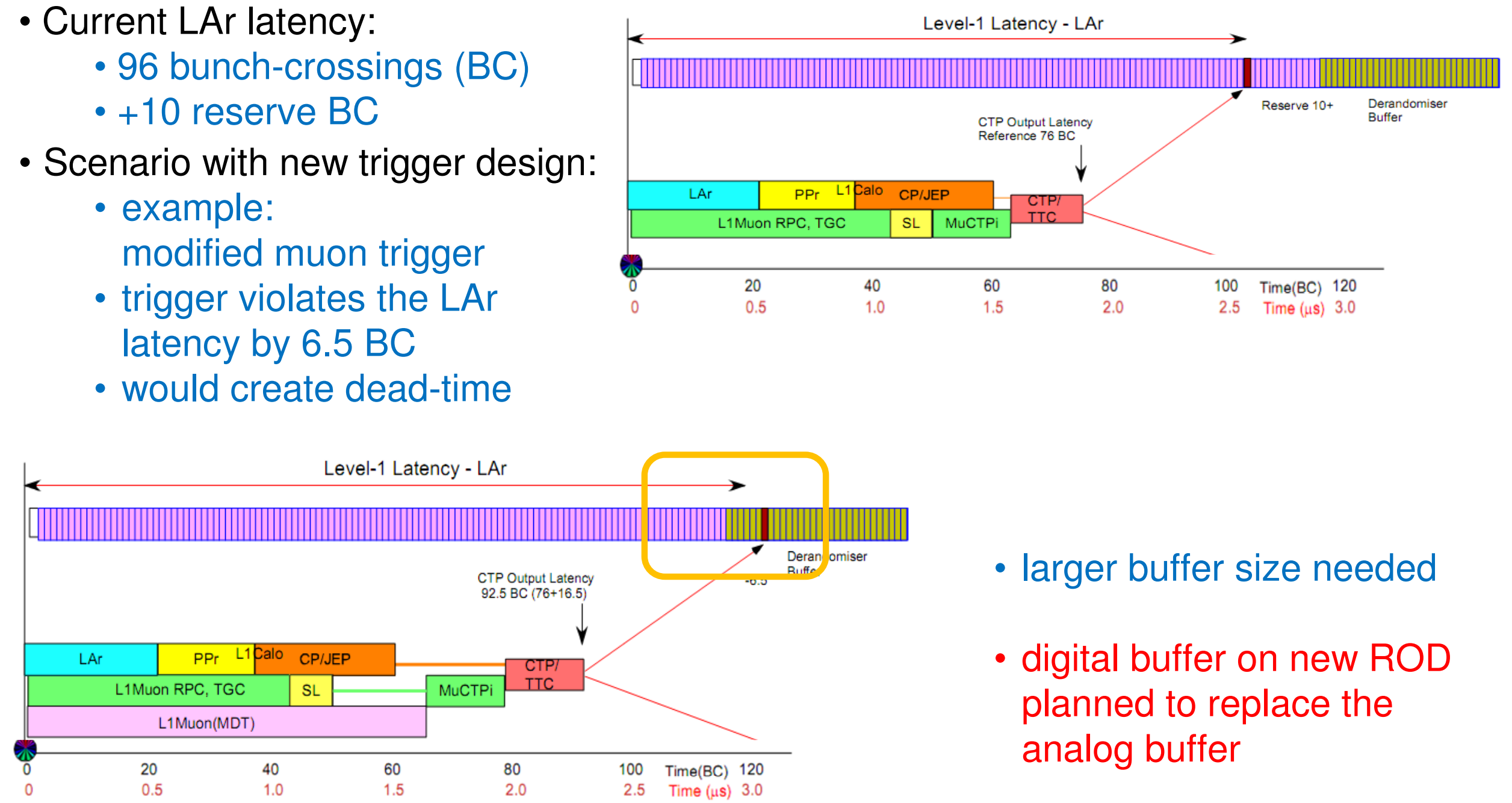

- larger buffer size needed

- digital buffer on new ROD planned to replace the analog buffer 


\section{ROD Input for High Granularity Trigger}

- current performance limitations:

- fixed analog trigger sums

- read-out and trigger upgrade at HL-LHC:

- ROD prepares digital data for hardware triggers

- higher granularity for better isolation of photons and electrons and pion tagging

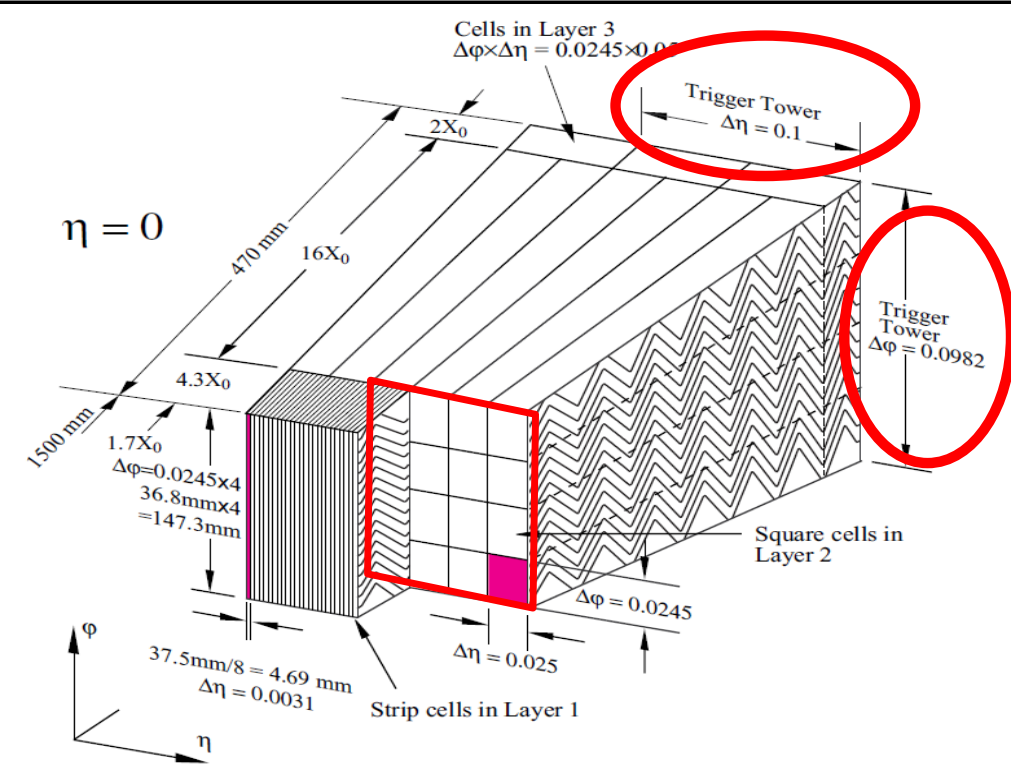

\begin{tabular}{|l|l|l|}
\hline & today & upgrade scenario \\
\hline hardware triggers & L1 & L0 and L1 \\
\hline trigger input & analog & digital \\
\hline trigger tower size in $\Delta \eta \times \Delta \varphi$ & $0.1 \times 0.1$ & up to $0.025 \times 0.025$ \\
\hline trigger tower depth & 4 layers summed & 4 layers separate \\
\hline data size / $0.1 \times 0.1$ tower & 16 bit & $\sim 200$ bit \\
\hline latency up to trigger input & $1.2 \mu$ s (L1) & $1.2 \mu$ s (L0) \\
\hline
\end{tabular}




\section{Requirements for New Hardware}

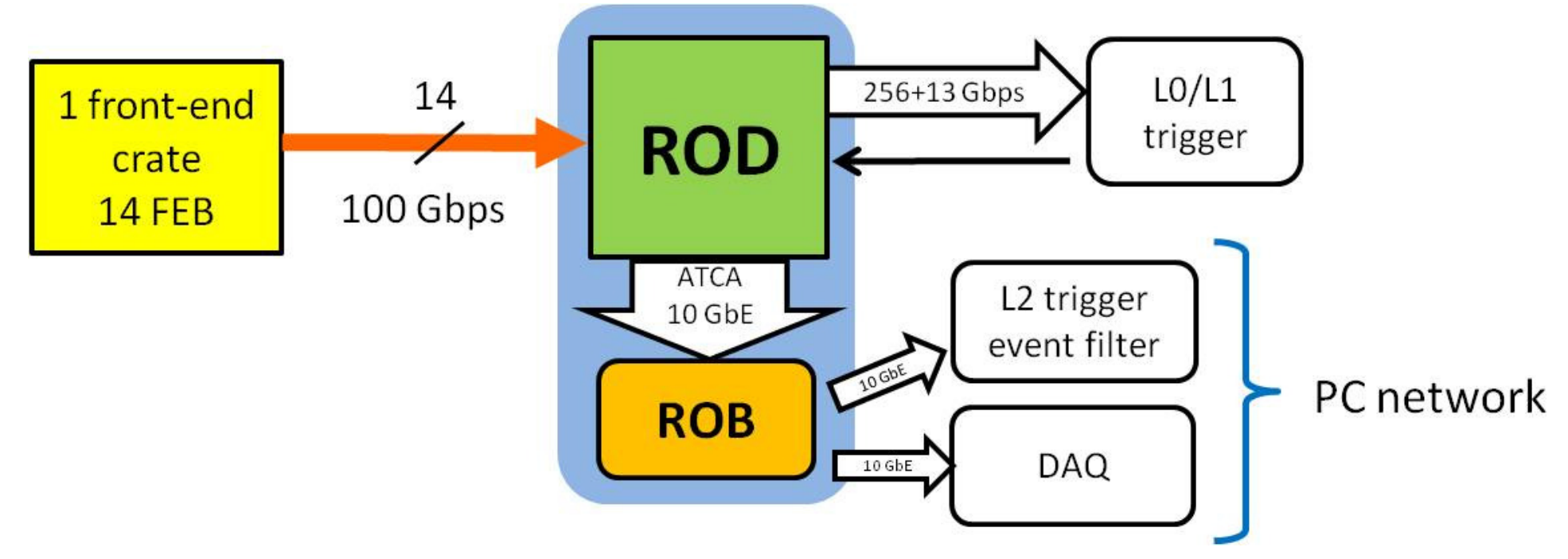

- Front-end electronics

- preamp, shaping, digitization of data @ BC rate $40 \mathrm{MHz}$ (no buffering anymore)

- high performance, radiation tolerant optical links needed for $100 \mathrm{Gbps}$ (see talk by A. Liu)

- Read-out driver (ROD)

- $40 \mathrm{MHz}$ input rate, connected to 14 front-end boards if possible

- digital calculation of energy, time, quality factor for each calorimeter cell

- perform L0/L1 sum digitally based on raw data $\rightarrow$ higher granularity, flexible

- send digitized data to L0 trigger @40 MHz

- local data buffer, send data to read-out buffer (ROB) after L1A @ $100 \mathrm{kHz}$ 


\section{Readout Scheme for HL-LHC}

- each ROD receives $14 \times 100 \mathrm{Gbit} / \mathrm{s}=\sim 1.4 \mathrm{Tbit} / \mathrm{s}$

- high speed parallel fiber optical transceiver (e.g.12 fibers @ 10Gbps)

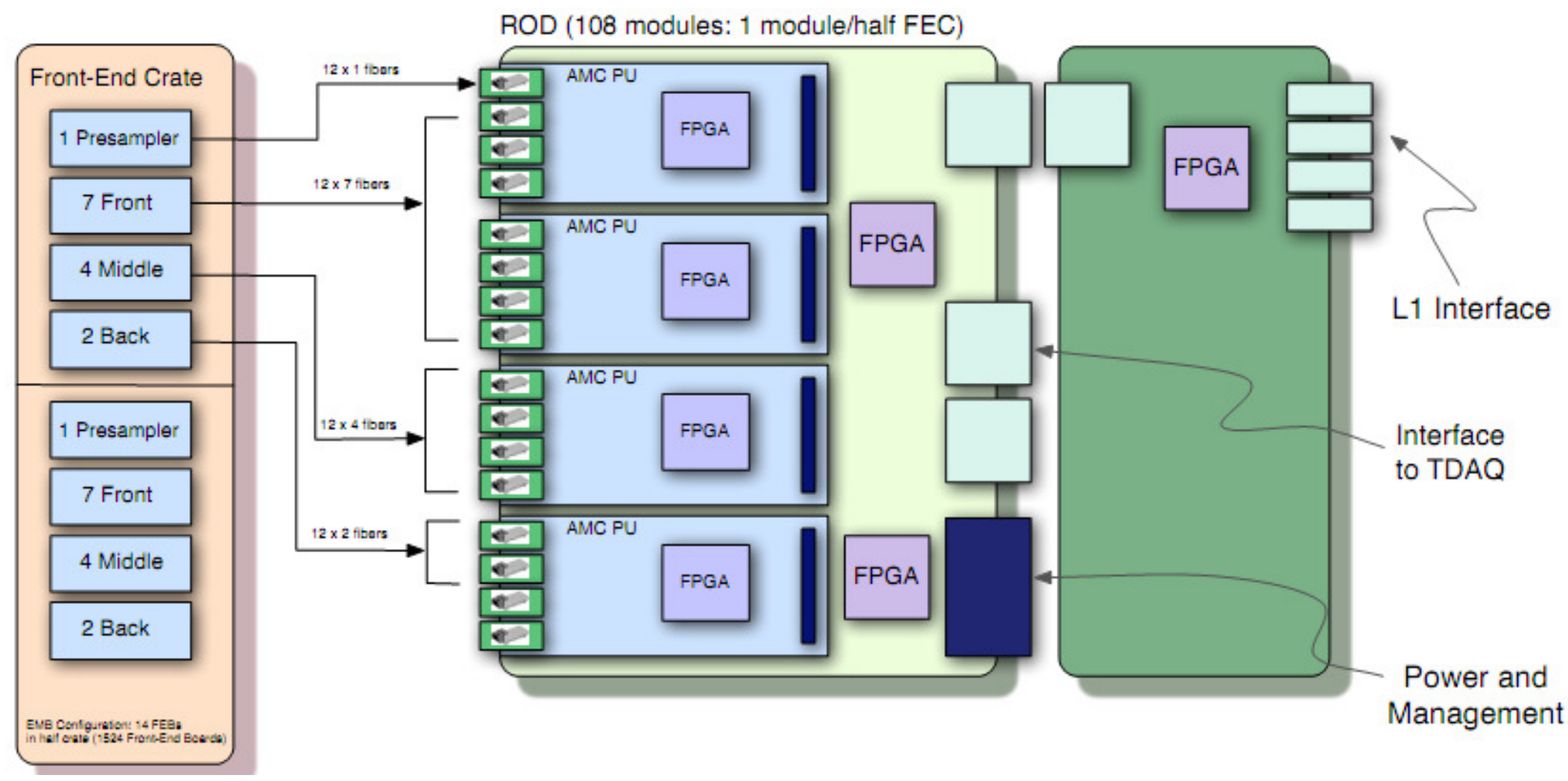

- ongoing ROD R\&D:

- use FPGAs for high performance SERDES

- use FPGAs for parallel data processing with high bandwidth

- follow up and explore technology evolution (FPGA, ATCA) 


\section{Sub-ROD + ROD Injector Development (BNL, Arizona)}

- Sub-ROD and ROD Injector design:

- ATCA form factor

- 75 Gbps fiber-optic link

- SNAP-12 parallel optical connector

- FPGA SERDES: Xilinx Virtex 5 FX on SubROD and Altera Stratix II GX on Injector

- Control and Power logic (IPMI, power conversion, hot plug, ...)

- Sub-ROD: standard design

- Injector: proprietary

- integration tests:

- ATCA power and control scheme functioning

- 6.25 Gbps per link reached $\rightarrow 75$ Gbps total

- used as platform for latency tests
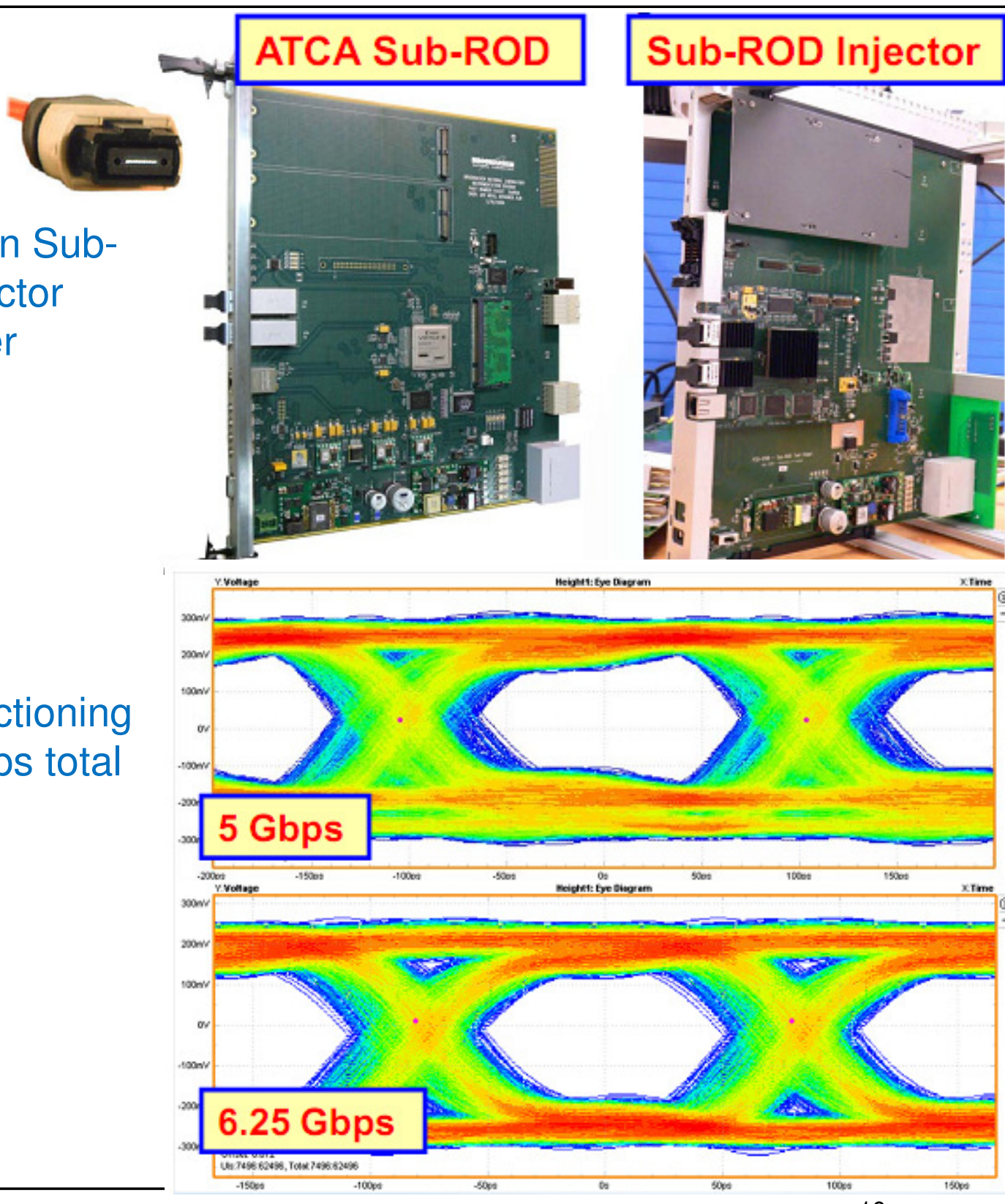

A Readout Driver for the ATLAS LAr Calorimeter at HL-LHC - Andy Kielburg-Jeka 


\section{Architecture of AMC Sub-ROD Processing Unit (BNL)}

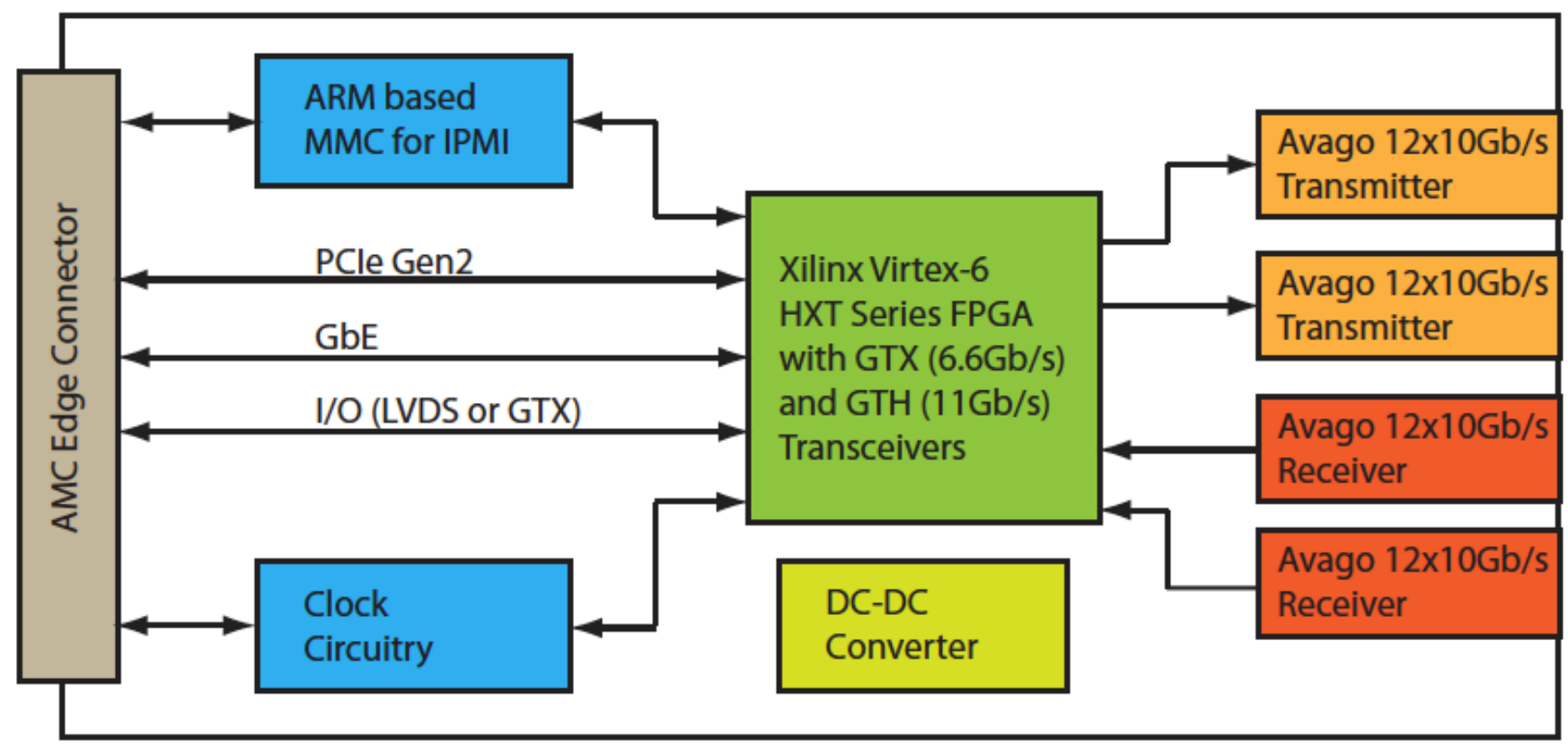

Block Diagram of AdvancedMC SubROD

- $\quad A M C$ format Sub-ROD Processing Unit can be tested in a low cost MicroTCA crate or on an ATCA carrier board

- $\quad M M C$ (Module Management Controller) is based on ARM processor for IPMI

- Precision clock circuitry and DC-DC converters for power management
- $\quad$ Based on high performance Xilinx Virtex-6 HXT series FPGA which has both GTX $(6.6 \mathrm{~Gb} / \mathrm{s})$ and $\mathrm{GTH}(11 \mathrm{~Gb} / \mathrm{s})$ transceivers

- High speed (12x10Gb/s) parallel fiber optical transceiver from Avago

- Versatile interface through AMC edge connector, PCle Gen2, GbE, LVDS and GTX 


\section{ROD Prototype in ATCA Format (LAPP, Annecy)}

- parallel development of ATCA based ROD board

- $10 \mathrm{~Gb}$ Ethernet is tested with commercial ATCA computing blades

- first design of proprietary ATCA board is ready

- R\&D goals:

- understand IPMI and power control

- develop control software framework

- on board communication between FPGAs

- full ROD demonstrator will use ALTERA Stratix IV FPGA

ROD Demonstrator

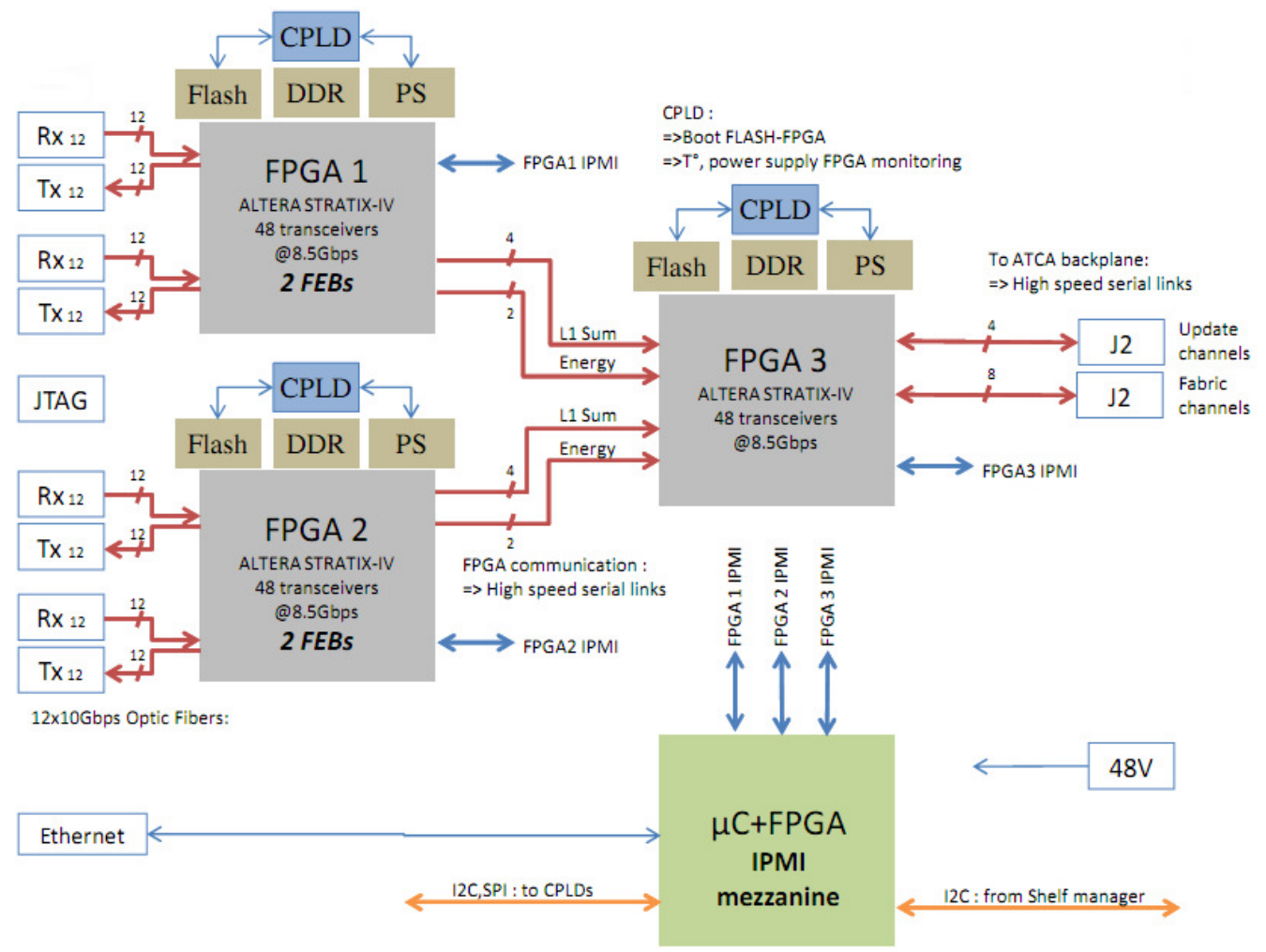




\section{ROD and ROB communication in ATCA System (TU Dresden)}

- read-out driver and read-out buffer test system:

- evaluate communication between ROD and ROB using $10 \mathrm{~Gb}$ Ethernet

- server PC is a ROB prototype

$\rightarrow$ planned to be a commercial ATCA blade in future

- hardware used:

- Radisys ATCA crate with dual star backplane

- 1 ATCA Sub-ROD equipped with Xilinx Virtex-5

- 24 x 10 Gbps switch with XFP transceiver

- optical $10 \mathrm{GbE}$ connection to server PC

- PC with dual Xeon 5550 CPU, 24 GB RAM, 4 Myricom 10 GbE dual port NICs

- ROB server performance reached:

-79 Gbps @ 30\% CPU load

- ROD prototype goals reached:

- 10 Gb XAUI interface implemented in Virtex-5 FPGA

- currently working on:

- $10 \mathrm{~Gb}$ Ethernet MAC in FPGA

- File system on PC-RAM via direct network access

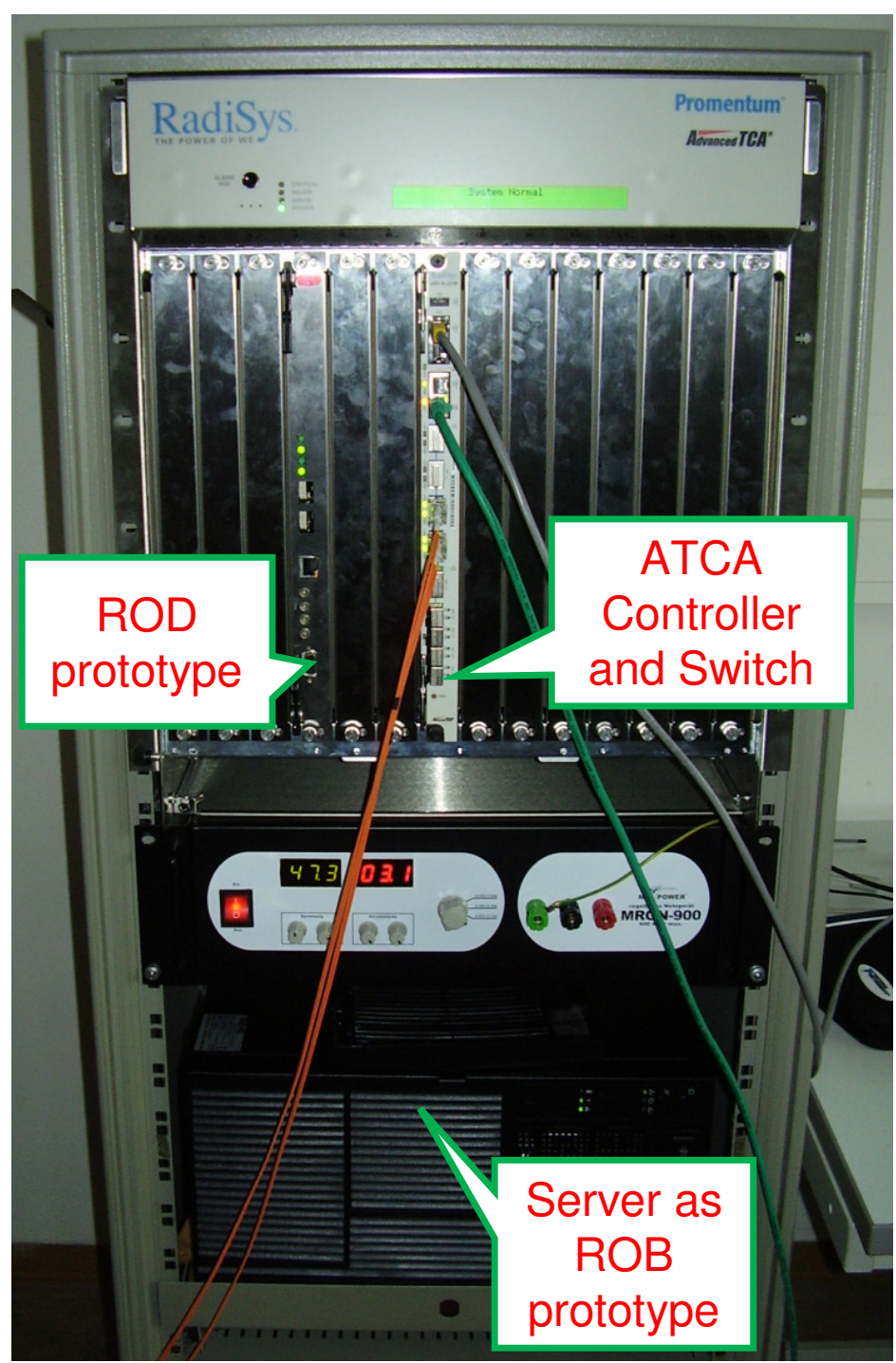




\section{Development of Digital FIR Filter in ROD FPGA}

- FIR filter prototype as core data processing unit

- incoming raw data

- 2 bit gain factor and 12 bit for energy $\rightarrow$ gg xx EEEE EEEE EEEE

- outgoing data: energy sum

$$
S=\sum_{i=1}^{5} a_{i}^{(\text {gain })} \cdot\left(E_{i}^{(\text {gain })}+\operatorname{Ped}_{i}^{(\text {gain })}\right)
$$

- 2 bit range factor and 14 bit for energy sum $\rightarrow$ rrs SSSS SSSS SSSS

- test hardware

- FPGA: Xilinx Virtes 5 XC5VFX70T

- testboard: Xilinx ML507

- Communication PC $\leftarrow \rightarrow$ FPGA:

- $1 \mathrm{GbE}, \mathrm{UDP} / \mathrm{IP}$

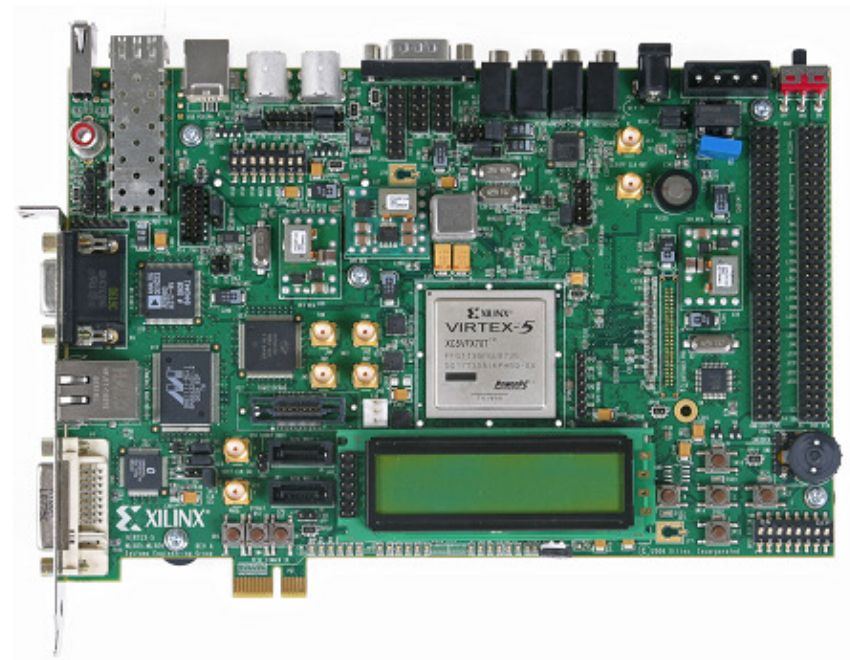




\section{ROD FPGA - Digital Filter Prototype}

- DSP modules used for energy sum calculation

- filter operates correctly up to $350 \mathrm{MHz}$

- filter internal latency is 3+2 clock cycles (due to DSP) $\rightarrow$ much less than 1 bunch-crossing (40 MHz)

- dynamic loading of calibration constants possible w/o FPGA firmware update

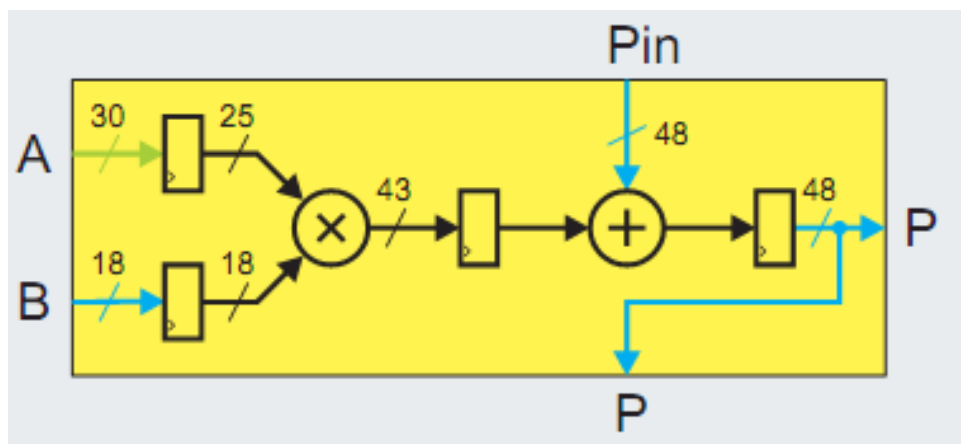

- Datasheet: DSPs can operate at up to $550 \mathrm{MHz} \rightarrow$ full speed not yet reached

- could process 12 interleaved channels

- 128 DSP slices allow implementation of 25 filter units $\rightarrow 2$ FEBs with 128 channels

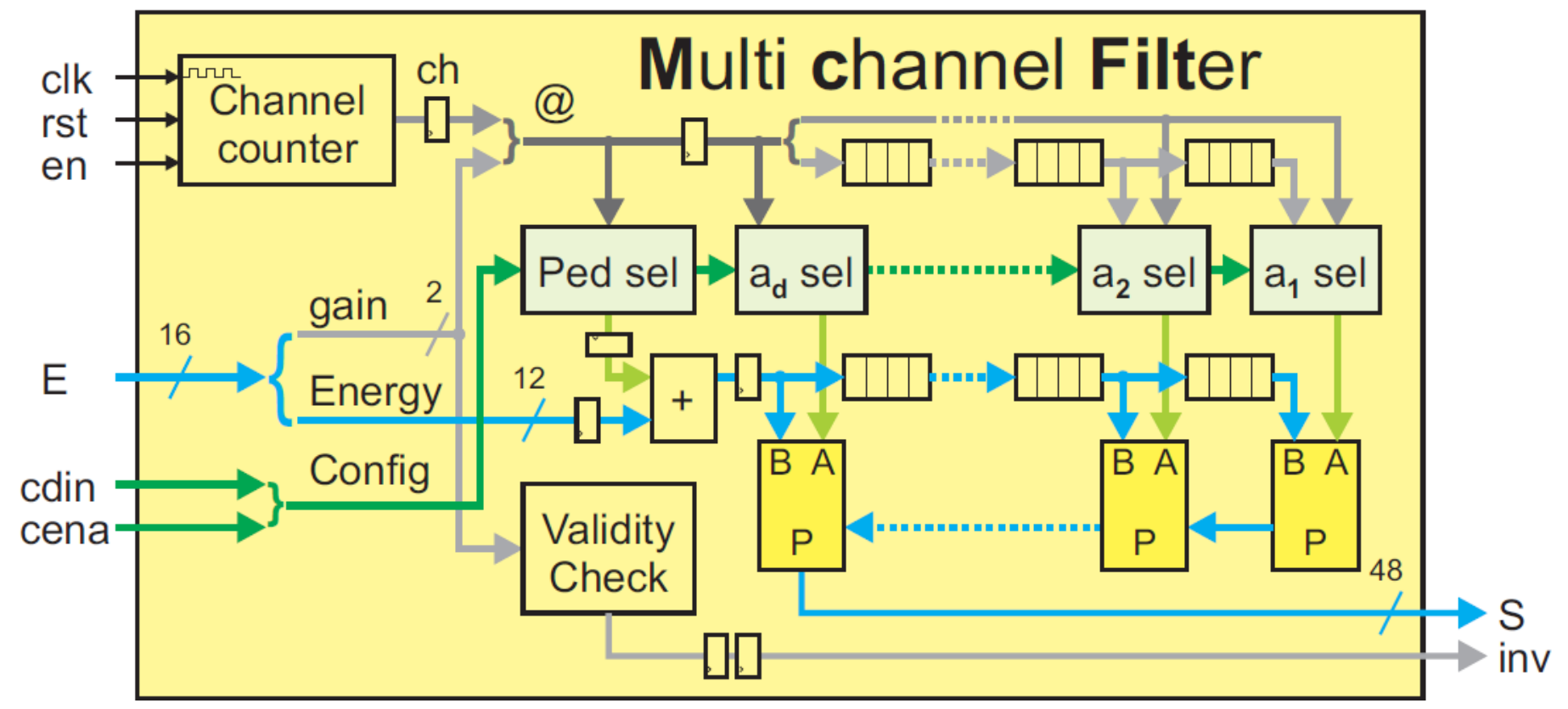




\section{Summary and Outlook}

- several reasons for updating / upgrading current LAr read-out environment

$\rightarrow$ a HL-LHC with advanced trigger logic with respect to granularity and flexibility

- new read-out scheme $\rightarrow$ change in functionality of FEB and ROD/ROB systems

- ROD planned to have additional capabilities to generate L0/L1 digital sums, perform optimal filtering based on digitized raw data and digital buffer of raw data

$\rightarrow$ first ROD prototypes in ATCA format

$\rightarrow$ SERDES test results with Xilinx/Altera FPGAs: 75 Gbps $\rightarrow 100$ Gbps required

$\rightarrow$ FIR energy filter: 4 channels at $350 \mathrm{MHz}$ in FPGA $\rightarrow$ need Nx128 at $500 \mathrm{MHz}$

$\rightarrow$ evaluate $10 \mathrm{GbE}$ communication to ROB

$\rightarrow$ further studies:

$\rightarrow$ event buffering and identification in FPGA

$\rightarrow$ evaluate integration of all tasks in FPGA(s) on ATCA ROD

$\rightarrow$ evaluate resource limits 


\section{Thank you for your attention!}




\section{LAr Calorimeter Signal}

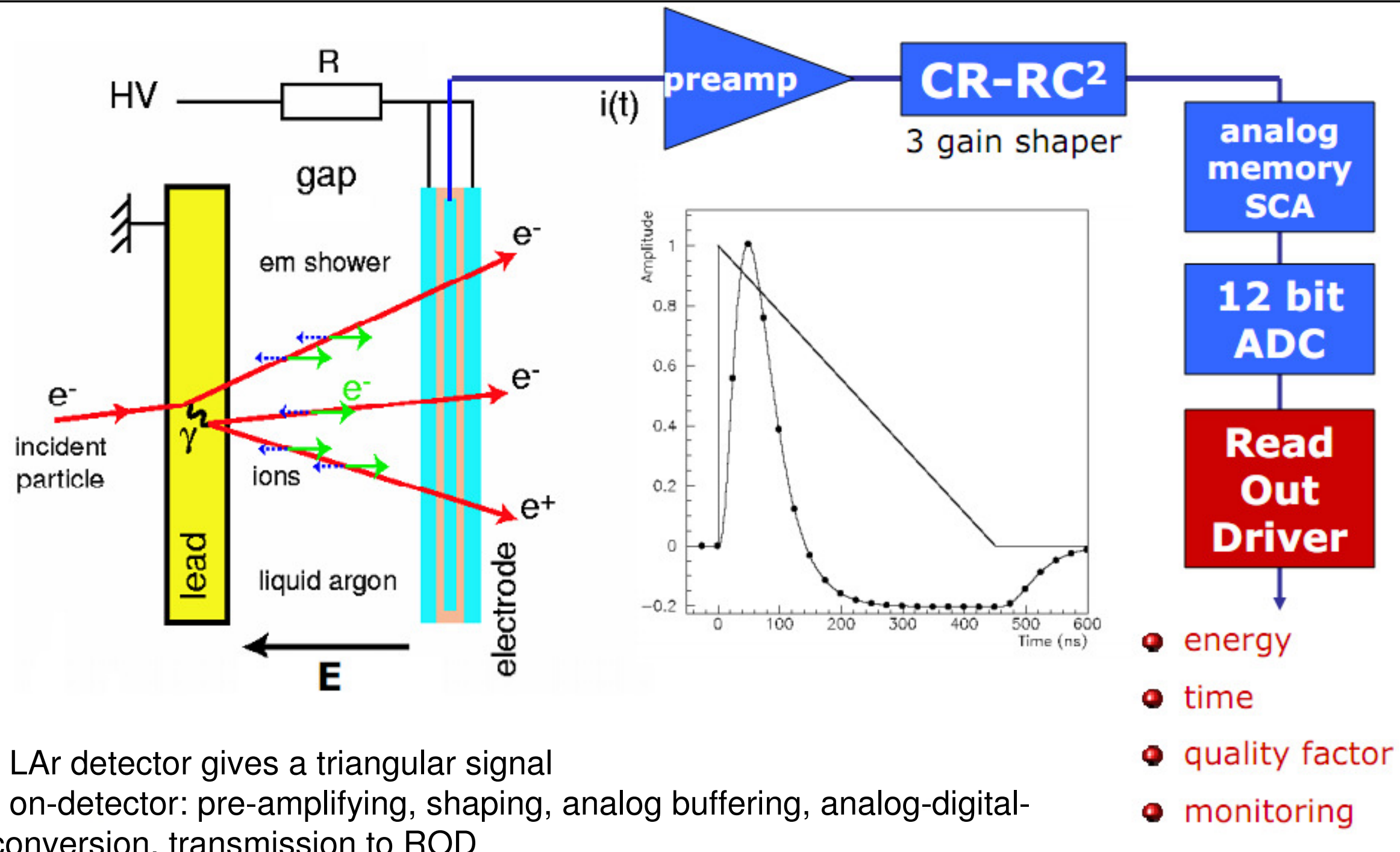

- off-detector: optimal filtering applied to obtain optimum $\mathrm{S} / \mathrm{N}$ ratio 


\section{Readout scheme for HL-LHC}

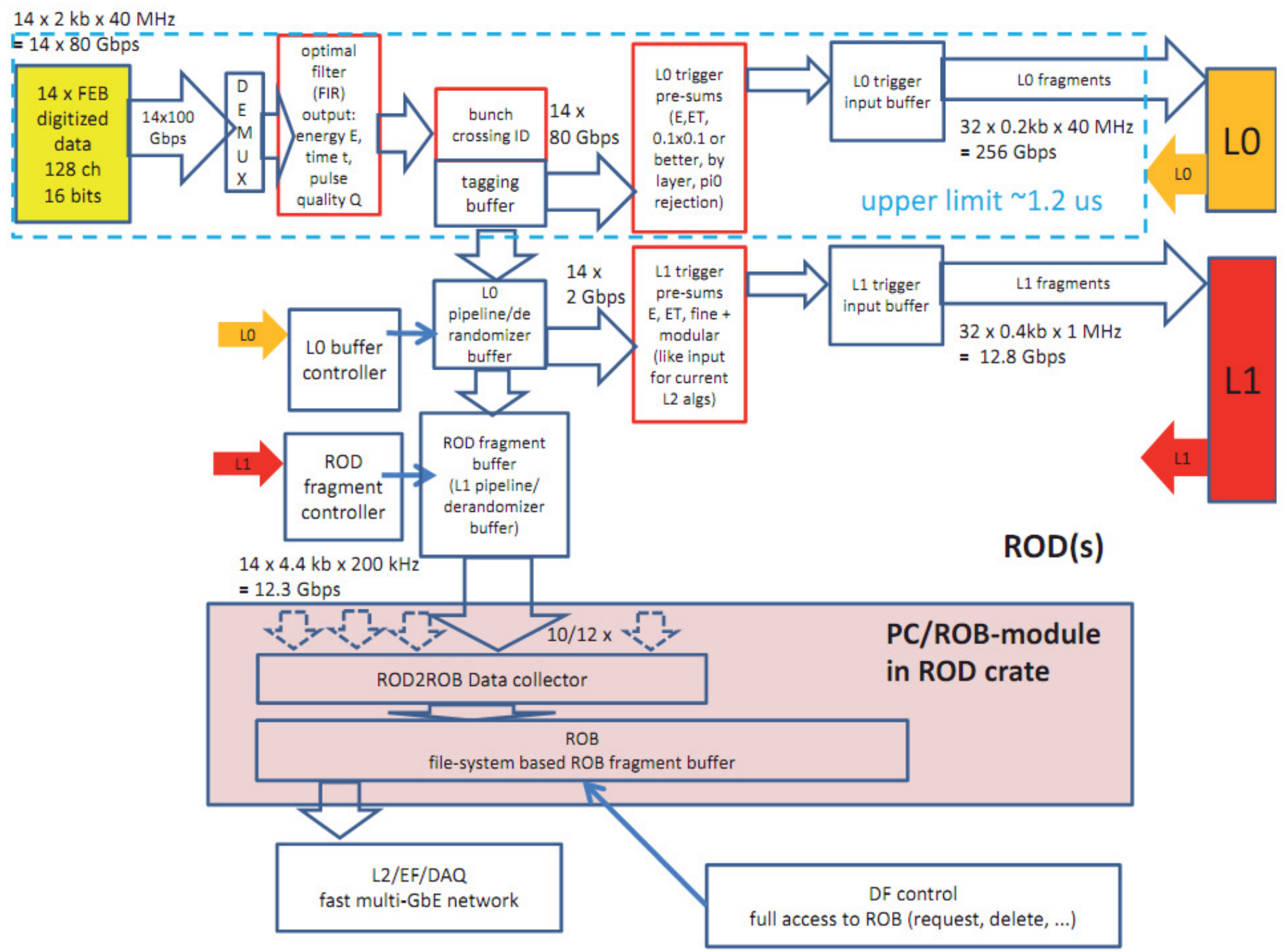




\section{ROB development: status + Outlook}

- read-out buffer: high-level-trigger and DAQ buffer (as today)

- ROD is planned to be $10 \mathrm{GbE}$ interface to ROB

- new:

- event data is stored as files directly in RAM of ROB (use proper file format)

- Idea: use of standard components and protocols (ATCA, commercial computing boards, 10GbE, UDP, ...)

- Status:

- Dual Xeon 5550 PC (4 cores, 6.4GT, 24GB RAM)

- 4 x Myricom 10GbE dual port NICs

- performance:

- 80Gbps @ 60\% CPU (9.9Gbps, MTU9000)

- HT on: 30\% CPU

- to be done:

- data transfer from

ROD FPGA to ROB PC

- TDAQ software tests

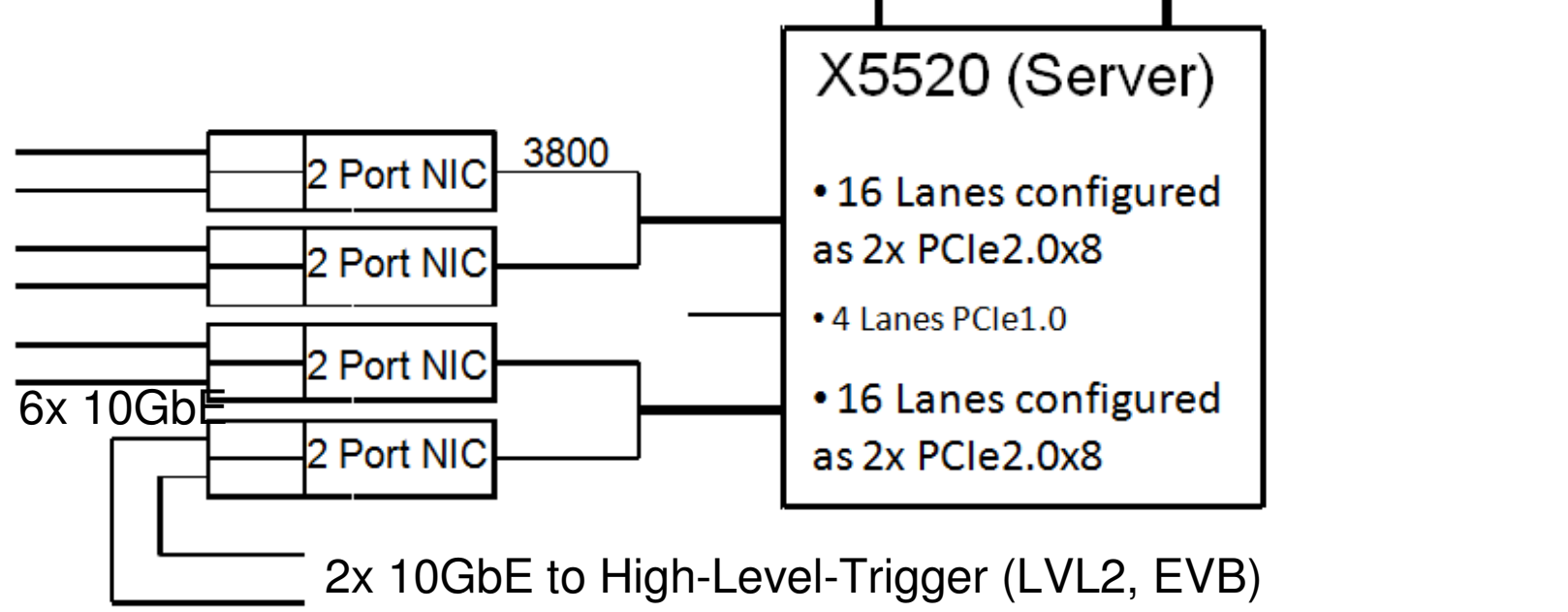




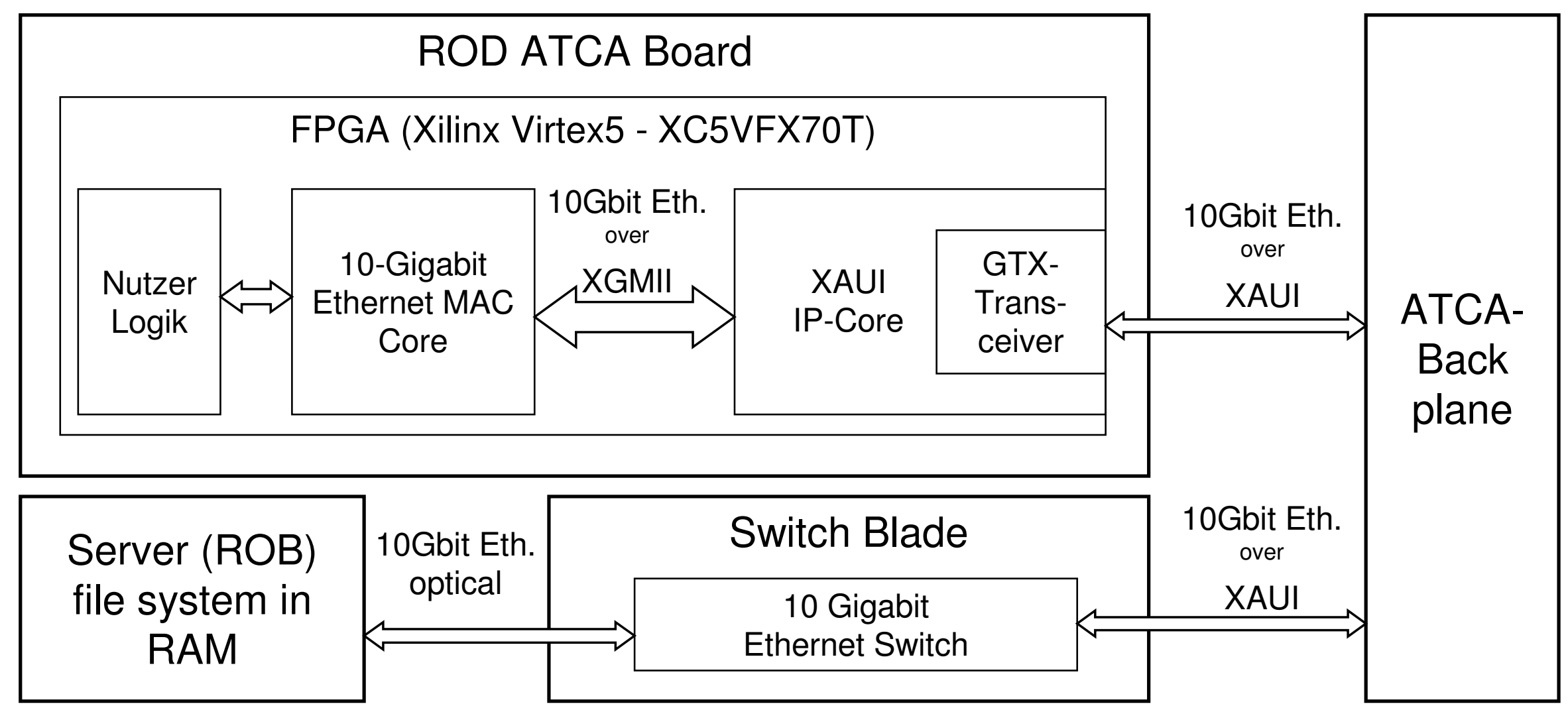

